\title{
High levels of centrosomal protein 55 expression is associated with poor clinical prognosis in patients with cervical cancer
}

\author{
JINGYI QI, GELIN LIU and FANG WANG \\ Department of Reproductive Medicine, Luoyang Central Hospital Affiliated to Zhengzhou University, \\ Luoyang, Henan 471000, P.R. China
}

Received June 7, 2016; Accepted March 7, 2018

DOI: $10.3892 / \mathrm{ol} .2018 .8448$

\begin{abstract}
Centrosomal protein 55 (CEP55) has been proposed to have a role in tumor development. However, the expression pattern and clinical relevance of CEP55 has, to the best of our knowledge, not yet been investigated in cervical cancer. The mRNA levels of CEP55 in cervical cancer tissues and paired adjacent non-cancerous tissues were examined by reverse transcription-quantitative polymerase chain reaction (RT-qPCR). The present study assessed the association between immunohistochemical staining of CEP55 and clinicopathological characteristics and survival rates of patients. Compared with the adjacent non-cancerous tissues, CEP55 expression was significantly increased in cervical tumor tissues, as demonstrated by the results of RT-qPCR. High expression of CEP55 was significantly associated with lymph node metastasis $(\mathrm{P}=0.008)$ and advanced tumor stage $(\mathrm{P}=0.010)$. Furthermore, CEP55 overexpression in cervical cancer specimens was significantly associated with poor 5 -year overall and recurrence-free survival rates $(\mathrm{P}=0.021$ and $\mathrm{P}=0.010$, respectively). The results of multivariate Cox regression analysis revealed that CEP55 expression was a significant, independent predictor for the survival of patients with cervical cancer (hazard ratio $=3.057 ; \mathrm{P}=0.035$ ). These data indicated that high CEP55 expression was associated with lymph node metastasis and was an independent predictive factor for an unfavorable prognosis in patients with cervical carcinoma.
\end{abstract}

\section{Introduction}

Cervical cancer is the third most common gynecological malignancy and one of leading causes of cancer-associated mortality in the world (1). Although current treatment strategies for cervical cancer utilize chemotherapy alongside

Correspondence to: Dr Jingyi Qi, Department of Reproductive Medicine, Luoyang Central Hospital Affiliated to Zhengzhou University, 288 M Zhongzhou Road, Luoyang, Henan 471000, P.R. China

E-mail: qijingyi729@sina.com

Key words: centrosomal protein 55, cervical cancer, prognosis surgical resection or radiotherapy, the survival rate of patients with late-stage cervical cancer remains poor (2). The tumor stage at diagnosis and tumor volume are the most notable prognostic factors for patients. However, regional lymph node involvement, distant metastasis and loco-regional recurrence occur frequently, despite the use of radical surgery and multimodal therapies (3). Metastasis is the primary cause of mortality from cervical cancer; however, the mechanism of metastasis in patients with cervical cancer is complicated and remains incompletely understood (4,5). Therefore, novel well-characterized biomarkers would aid clinicians in predicting metastatic progression and the prognosis of patients with cervical cancer.

Centrosomal protein 55 (CEP55) is a microtubule-bundling protein (6). CEP55 was originally identified as a novel coiled-coil protein during bioinformatic screening for genes involved in mitosis (7). The CEP55 gene is located on chromosome 10q23.33 and encodes three central coiled-coil domains. As a mitotic phosphoprotein, CEP55 is recruited to the midbody during cytokinesis and is essential for the completion of cell division (8). The upregulation or downregulation of CEP55 can result in cytokinesis defects and an increase in the number of multinucleated cells. Evidence indicates that CEP55 is specifically expressed in the normal human testes and in various malignancies; it can regulate membrane fission and fusion events near the midbody ring at the last stage of cancer cell division $(9,10)$. Overexpression of CEP55 increased the growth of cancer cells, indicating that the suppression of CEP55-mediated signal transduction may be a novel therapeutic approach for a range of human tumor types (11). In addition, elevated CEP55 expression in mammalian cells can enhance cellular migration and invasion, again indicating its potential as a therapeutic target for treating patients with cancer (12). The results of functional assays indicated that CEP55 displays a number of characteristics associated with oncogenic behavior, including anchorage-independent growth, enhanced proliferation at low serum levels and the in vivo induction of tumorigenesis (13). However, the functional role of CEP55 in cervical cancer remains largely unknown.

The present study aimed to investigate the expression pattern of CEP55 in cervical cancer, and its clinicopathological implications. The current study provides evidence that CEP55 expression was significantly increased in cervical cancer tissues, compared with adjacent non-cancerous 
tissues. High CEP55 expression was observed in $62.0 \%$ of patients (77/124) with cervical cancer, and was significantly associated with lymph node metastasis and advanced tumor stage. Furthermore, high CEP55 expression was a significant, independent predictive factor for survival of patients with cervical cancer.

\section{Materials and methods}

Patients and samples. For the present retrospective study, archived formalin-fixed, paraffin-embedded tissue specimens from 124 patients with primary cervical cancer who underwent radical hysterectomy at Luoyang Central Hospital affiliated to Zhengzhou University between December 2004 and December 2007 were recruited. For the reverse transcription-quantitative polymerase chain reaction (RT-qPCR) assay, fresh cervical cancer and paired adjacent non-cancerous tissue samples were obtained from eight patients with cervical cancer who underwent surgical resection. Tumor resection was performed by experienced surgeons, and the surgical procedures, which followed the International Federation of Gynecology and Obstetrics (FIGO) guidelines (14), were similar in all patients who underwent radical resection. No patients had received chemotherapy or radiotherapy prior to surgery. The median age of the cohort was 44 years (range, 25-68 years). All patients were staged using the FIGO staging system. Tissue samples from non-cancerous cervical lesions were also collected during the study period and served as the normal controls. Written informed consent was obtained from each patient for the use of the tissue samples for research purposes. The present study was approved by the Institutional Ethics Committee of the Luoyang Central Hospital affiliated to Zhengzhou University (Zhengzhou, China).

$R T-q P C R$. Total RNA was extracted from cervical cancer tissues and adjacent non-cancerous tissues using TRIzol reagent (Invitrogen; Thermo Fisher Scientific, Inc., Waltham, MA, USA) according to the manufacturer's protocol. RNAse-free DNAase I was used to eliminate DNA contamination. Following reverse transcription of the total RNA using cDNA synthesis kit (Thermo Fisher Scientific, Inc.), the first-strand cDNA was then used as the template for detection of CEP55 expression by RT-qPCR with the SYBR-Green I chemistry using SYBR-Green PCR kit (Applied Biosystems; Thermo Fisher Scientific, Inc.). Thermocycling begins with $95^{\circ} \mathrm{C}$ for 5 min before 40 cycles of amplification at $95^{\circ} \mathrm{C}$ for $15 \mathrm{sec}$, and $60^{\circ} \mathrm{C}$ for $60 \mathrm{sec}$. GAPDH was used as internal control. The primers were as follows: CEP55 forward, 5'-TGAAGAGAA AGACGTATTGAAACAA-3' and reverse, 5'-GCAGTTTGG AGCCACAGTCT-3'; and GAPDH forward, 5'-AGCCACATC GCTCAGACAC-3' and reverse, 5'-GCCCAATACGACCAA ATCC-3'. The relative expression level was determined using the $2^{-\Delta \Delta C q}$ method (15). Data are presented as the expression level relative to the calibrator (control sample).

Immunohistochemistry. Immunohistochemical analysis was performed to investigate CEP55 expression in 124 paraffin-embedded tissues, which had been processed into 5 -mm serial sections. Briefly, the sections were de-waxed in xylene and rehydrated in a graded alcohol series $(95,80,70$
Table I. Association between CEP55 expression and clinicopathological variables of 124 patients with cervical cancer.

\begin{tabular}{lrrrr}
\hline & & \multicolumn{2}{c}{ CEP55 } & \\
Parameter & Total & Low & High & P-value \\
\hline Age, years & & & & 0.194 \\
$\leq 50$ & 75 & 25 & 50 & \\
$>50$ & 49 & 22 & 27 & \\
FIGO stage & & & & 0.010 \\
$\quad$ IB & 83 & 38 & 45 & \\
$>$ IB & 41 & 9 & 32 & \\
Tumor size, cm & & & & 0.713 \\
$\leq 4$ & 90 & 35 & 55 & \\
$>4$ & 34 & 12 & 22 & \\
Differentiation & & & & 0.731 \\
$\quad 1 / 2$ & 97 & 36 & 61 & \\
3 & 27 & 11 & 16 & \\
Histological type & & & & 0.310 \\
$\quad$ SCC & 85 & 41 & 44 & \\
$\quad$ AC & 39 & 15 & 24 & \\
LN metastasis & & & & 0.008 \\
$\quad$ No & 95 & 53 & 42 & \\
$\quad$ Yes & 29 & 8 & 21 & \\
\hline
\end{tabular}

CEP55, centrosomal protein 55; FIGO, International Federation of Gynecology and Obstetrics; SCC, squamous cell carcinoma; AC, adenocarcinoma; LN, lymph node.

and $50 \%, \mathrm{v} / \mathrm{v}$ ), then they were boiled for $3 \mathrm{~min}$ in $10 \mathrm{mmol} / \mathrm{l}$ of citrate buffer ( $\mathrm{pH}$ 6.0) for antigen retrieval. Following the inhibition of endogenous peroxidase activities for $15 \mathrm{~min}$ at room temperature by $3 \%$ hydrogen peroxide in methanol, slides were treated with $1 \%$ bovine serum albumin (Gibco; Thermo Fisher Scientific, Inc.) for $30 \mathrm{~min}$ at room temperature to block non-specific binding. Next, the slides were incubated with a rabbit monoclonal anti-CEP55 antibody (1:100; Abcam, Cambridge, UK; cat. no. ab214302) overnight at $4^{\circ} \mathrm{C}$. Following washing with $0.01 \mathrm{M}$ phosphate buffer, the tissue sections were then incubated with the biotinylated secondary antibody (goat anti-rabbit; dilution, 1:1,000; Abcam; cat. no. ab6702) at room temperature for $30 \mathrm{~min}$, followed by further incubation with streptavidin-horseradish peroxidase complex (OriGene Technologies, Inc., Rockville, MD, USA) for $20 \mathrm{~min}$ at room temperature. Negative control slides were prepared by omitting the primary antibody under the same experimental conditions, and the absence of non-specific immunoreactive staining was confirmed.

Semi-quantitative estimation of expression was made using a composite score obtained by multiplying the values of staining intensity and relative abundance of positive cells by two independent pathologists from Luoyang Central Hospital affiliated to Zhengzhou University using a transmitted light microscope (x400). The intensity of staining was graded as follows: 0 , no staining; 1 , weak staining; 2 , moderate staining; or 3 , strong staining. The abundance of positive cells was 
Table II. Cox regression analyses for predictors of outcome.

\begin{tabular}{|c|c|c|c|c|}
\hline \multirow[b]{2}{*}{ Prognostic variable } & \multicolumn{2}{|c|}{ Overall survival } & \multicolumn{2}{|c|}{ Recurrence-free survival } \\
\hline & Hazard ratio $(95 \% \mathrm{CI})$ & P-value & Hazard ratio $(95 \% \mathrm{CI})$ & P-value \\
\hline Age (>50 vs. $\leq 50$ years $)$ & $1.580(0.149-4.977)$ & 0.413 & $1.385(0.109-4.111)$ & 0.502 \\
\hline Tumor stage (>IB vs. IB) & $2.492(0.995-5.114)$ & 0.004 & $2.247(0.713-6.410)$ & 0.042 \\
\hline Tumor size ( $>4$ vs. $\leq 4 \mathrm{~cm}$ ) & $2.256(0.612-5.102)$ & 0.074 & $2.197(0.710-5.016)$ & 0.052 \\
\hline Tumor grade (3 vs. 1/2) & $1.247(0.210-2.515)$ & 0.401 & $1.295(0.265-2.258)$ & 0.550 \\
\hline Histological type (SCC vs. AC) & $1.813(1.555-4.211)$ & 0.214 & $1.368(1.118-4.764)$ & 0.613 \\
\hline LN metastasis (yes vs. no) & $4.258(1.819-7.314)$ & 0.026 & $3.349(1.201-7.594)$ & 0.014 \\
\hline CEP55 expression (high vs. low) & $3.057(1.404-7.482)$ & 0.035 & $3.267(1.406-4.594)$ & 0.065 \\
\hline
\end{tabular}

CI, confidence interval; SCC, squamous cell carcinoma; AC, adenocarcinoma; LN, lymph node; CEP55, centrosomal protein 55.

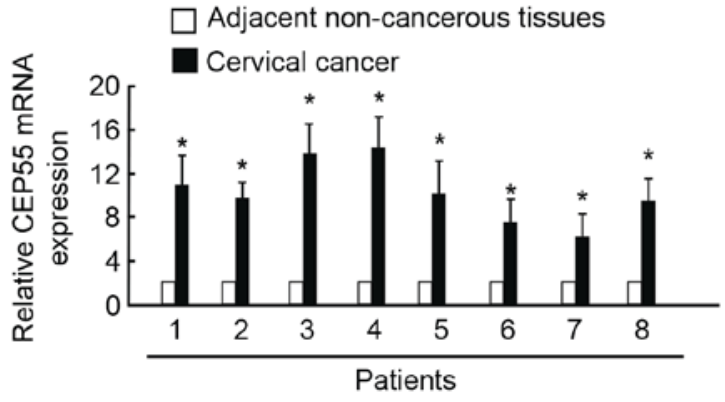

Figure 1. Expression of CEP55 is upregulated in cervical cancer tissues compared with non- tumorous tissues by reverse transcription-quantitative polymerase chain reaction. ${ }^{*} \mathrm{P}<0.05$. CEP55, centrosomal protein 55.

graded from 0 to 4 , as follows: $0,<5 \%$ positive cells; $1,5-25 \%$; $2,26-50 \% ; 3,51-80 \%$; and 4, >80\%). The composite score was defined as low expression for a score of 0-4 and as high expression for a score of 6-12.

Statistical analysis. Several clinicopathological factors were evaluated, including age, FIGO stage, tumor differentiation and lymph node status. The $\chi^{2}$ test was used to evaluate the association between clinicopathological variables and expression of CEP55. $\mathrm{P}<0.05$ was considered to indicate a statistically significant difference. Clinicopathological variables and CEP55 expression were taken into account for the survival analysis, based on the Kaplan-Meier method, with statistical significance assessed using the log-rank test. To determine the effect of particular prognostic factors on survival, a multivariate analysis was performed according to the Cox regression model.

\section{Results}

CEP55 was overexpressed in cervical cancer specimens. To determine whether CEP55 participates in the pathogenesis of cervical carcinoma, RT-qPCR was performed on eight pairs of cervical cancer and adjacent non-cancerous tissue samples. CEP55 expression was higher in the tumor specimens than in the normal tissues. The mean expression level of CEP55 was 9 -fold higher in cervical cancer samples than in normal tissue samples (Fig. 1). Representative immunostaining for CEP55 in cervical cancer tissues is depicted in Fig. 2.

Clinicopathological factors and survival rates of patients with cervical cancer expressing CEP55. To investigate whether an increase in the expression of CEP55 was associated with various prognostic factors, patients were classified into groups based on the semi-quantitative results of immunohistochemical staining (low vs. high CEP55 expression). As depicted in Table I, patients with an advanced tumor stage and a higher rate of lymph node metastasis expressed significantly higher levels of CEP55 than patients with early stage tumors $(\mathrm{P}=0.010)$ or no lymph node metastasis $(\mathrm{P}=0.008)$. No significant difference between levels of CEP55 expression was observed in patients with different ages or histological disease subtypes at the time of diagnosis.

Survival analysis using the Kaplan-Meier method revealed that the prognosis of patients with tumors expressing high levels of CEP55 was significantly poorer than those with tumors expressing low levels of CEP55 $(\mathrm{P}=0.021$ and $\mathrm{P}=0.010$ for overall and recurrence-free survival, respectively) (Fig. 3). Multivariate analysis revealed that CEP55 expression was a significant independent prognostic factor for the overall survival rate of patients ( $\mathrm{P}=0.035)$, as depicted in Table II. The results of the present study clearly indicated that the prognosis of patients with cervical cancer was associated with CEP55 expression.

\section{Discussion}

Cervical cancer is one of the most common malignancies in females worldwide, culminating in 265,700 deaths in 2012 (16). EP55 is involved in tumorigenesis in a number of types of cancer. However, the expression pattern of CEP55 has, to the best of our knowledge, not been investigated in cervical cancer. The current study revealed that CEP55 expression was significantly higher in cervical cancer tissues than in adjacent non-cancerous tissues, as evidenced by the results of RT-qPCR. High CEP55 expression was observed in $62.0 \%$ (77/124) tissues from patients with cervical cancer, and was significantly associated with lymph node metastasis and FIGO stage. Furthermore, the data from the present study indicated 

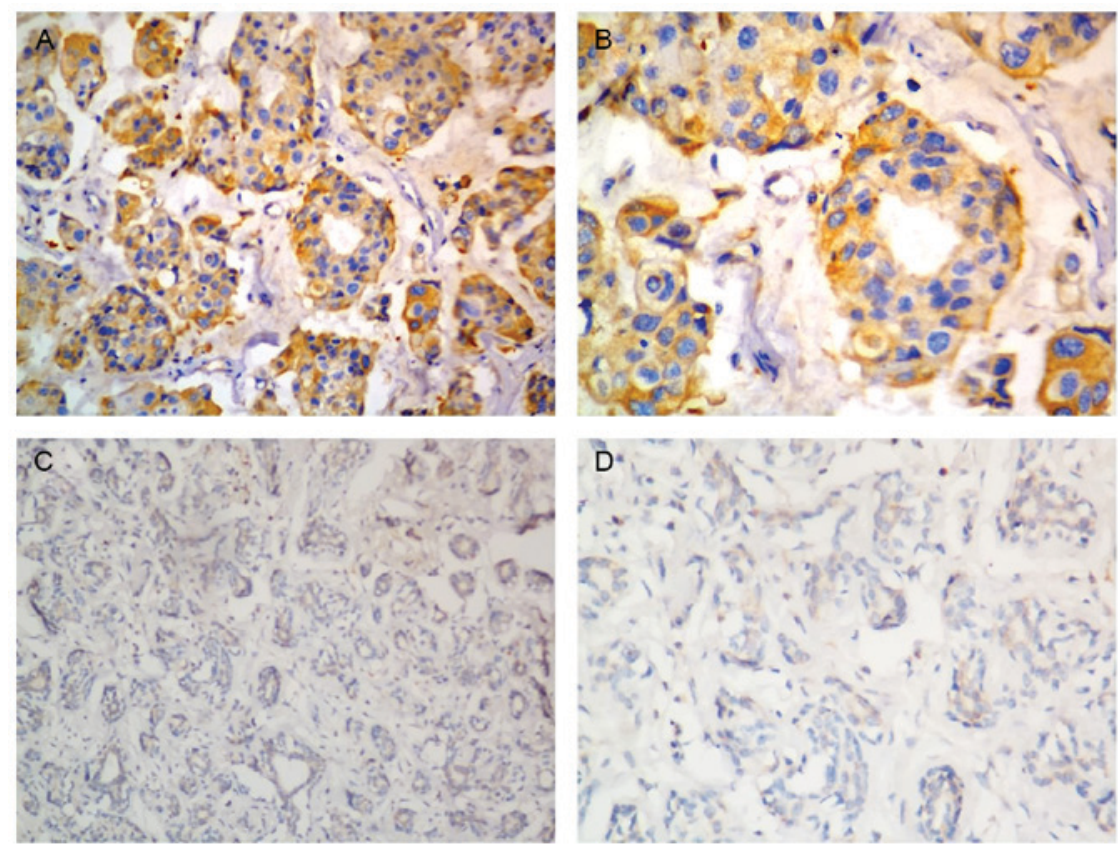

Figure 2. Representative immunostaining for centromere protein 55 expression in cervical cancer. (A) High expression, x200. (B) High expression, $\mathrm{x} 400$. (C) Low expression, $\mathrm{x} 200$. (D) Low expression, $\mathrm{x} 400$.
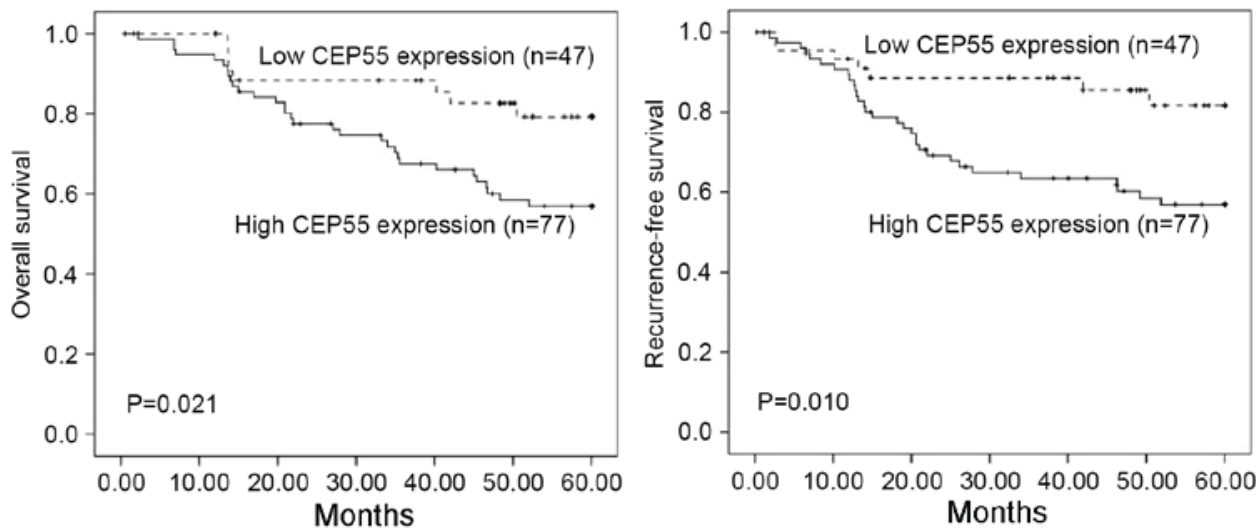

Figure 3. Kaplan-Meier survival curves for overall and recurrence-free survival rates, stratified by CEP55 expression. CEP55, centrosomal protein 55 .

that CEP55 expression was of significant, independent predictive value for the survival rates of patients with cervical cancer. Thus, the results of the current study also indicated that CEP55 could be a putative biomarker for tumor metastasis and patient prognosis in cervical cancer.

The centrosome is an organelle that has several key functions in the vertebrate cell cycle as the primary microtubule-organizing center. In tumor cells, a number of structural changes, including increases to the volume and number of centrosomes, the presence of supernumerary centrioles, the accumulation of excess pericentriolar material and the non-phosphorylation of centrosomal proteins (17). The centrosome-associated protein CEP55 is included in the pericentriolar material and, when overexpressed, can cause ectopic microtubule nucleation (18). Previous studies have revealed that CEP55 is overexpressed in several types of cancer, including hepatocarcinoma, colon carcinoma, ovarian carcinoma and lung cancer $(11-13,19)$. To the best of our knowledge, the current study is the first to demonstrate CEP55 was upregulated in cervical cancer tissues compared with the adjacent non-cancerous tissues. Taken together, the results of the current study indicated that CEP55 may have an oncogenic role in the development and progression of tumors.

Notably, CEP55 has been demonstrated to be involved in growth-associated signaling pathways in tumor cells. Chen et al (13) revealed that the cellular transformation driven by CEP55 is mediated by activation of the phosphoinositide 3-kinase/protein kinase B (AKT) pathway in hepatocellular carcinoma cells. Additionally, CEP55 expression levels were positively associated with increased cellular proliferation and that tumor protein p53 (hereafter p53) is able to negatively regulate the activity of the CEP55 promoter and protein, and that the existence of a p53/polo-like kinase 1/CEP55 axis in which p53 negatively regulates expression of CEP55, is a positive regulator of CEP55 protein stability (20). Additionally, Tao et al (21) demonstrated that the ectopic overexpression of 
CEP55 enhanced the cellular proliferation, colony formation and tumorigenicity of gastric cancer cells, which was mediated by the AKT signaling pathway and contributes to carcinogenesis and progression of gastric carcinogenesis. In the cohort assessed in the present study, CEP55 protein expression was significantly associated with the FIGO stage of patients with cervical cancer, further indicating that CEP55 expression was positively associated with the growth of cancer cells.

Several studies have assessed the role of CEP55 in tumor progression. Chen et al (22) observed that CEP55 expression was associated with increased aggressiveness of oral cavity squamous cell carcinoma by inducing the migratory and invasive behavior of cells via increased forkhead box M1 and matrix metalloproteinase- 2 activity. Further study by the same group revealed that CEP55 expression was associated with tumor progression in nasopharyngeal carcinoma and contributed to nasopharyngeal cell proliferation and metastasis via the osteopontin/cluster of differentiation 44 pathway (23). Hwang et al (24) demonstrated that fibulin-5 promoted nasopharyngeal carcinoma cell metastasis via the CEP55/AKT pathway and was associated with poor patient prognosis. In concordance with the findings of these prior studies, the current study demonstrated that CEP55 expression was significantly associated with lymph node metastasis in patients with cervical cancer. Notably, high CEP55 expression was an indicator of poor overall and recurrence-free survival rates in patients with cervical cancer.

In conclusion, higher expression of CEP55 in cervical carcinoma tissues was associated with lymph node metastasis and poor patients prognosis. CEP55 has the potential to be used as a therapeutic target for patients with cervical carcinoma and should be investigated further.

\section{Acknowledgements}

Not applicable.

\section{Funding}

The present study was supported by the Innovation Fund of Luoyang Central Hospital affiliated to Zhengzhou University (grant no. 20120420).

\section{Availability of data and materials}

The datasets used and/or analyzed during the current study are available from the corresponding author on reasonable request.

\section{Authors' contributions}

JQ participated in the data collecting and drafted the manuscript. GL performed the statistical analysis. JQ and FW participated in the design of the study. All authors analyzed and interpreted the patient data.

\section{Ethics approval and consent to purticipate}

The present study was approved by the Institutional Ethics Committee of the Luoyang Central Hospital affiliated to Zhengzhou University (Zhengzhou, China).

\section{Consent for publication}

Written informed consent was obtained from each patient for the use of the tissue samples for paper publication.

\section{Competing interests}

The authors declare that they have no competing interests.

\section{References}

1. Colombo N, Carinelli S, Colombo A, Marini C, Rollo D and Sessa C; ESMO Guidelines Working Group: Cervical cancer: ESMO Clinical Practice Guidelines for diagnosis, treatment and follow-up. Ann Oncol 23 (Suppl 7): vii27-vii32, 2012.

2. Tao X, Hu W, Ramirez PT and Kavanagh JJ: Chemotherapy for recurrent and metastatic cervical cancer. Gynecol Oncol 110 (3 Suppl 2): S67-S71, 2008.

3. Bellmunt J, Orsola A, Leow JJ, Wiegel T, De Santis M and Horwich A; ESMO Guidelines Working Group: Bladder cancer: ESMO Practice Guidelines for diagnosis, treatment and follow-up. Ann Oncol 25 (Suppl 3): iii40-48, 2014.

4. Galloway TJ and Ridge JA: Management of squamous cancer metastatic to cervical nodes with an unknown primary site. J Clin Oncol 33: 3328-3337, 2015.

5. Grossman JS: Management of metastatic cervical cancer: Should we ignore lessons learned from treating other advanced squamous cell cancers? J Clin Oncol 33: 965-966, 2015.

6. Doxsey S, McCollum D and Theurkauf W: Centrosomes in cellular regulation. Annu Rev Cell Dev Biol 21: 411-434, 2005.

7. Doxsey SJ: Molecular links between centrosome and midbody. Mol Cell 20: 170-172, 2005.

8. Fabbro M, Zhou BB, Takahashi M, Sarcevic B, Lal P, Graham ME, Gabrielli BG, Robinson PJ, Nigg EA, Ono Y and Khanna KK: Cdk1/Erk2- and Plk1-dependent phosphorylation of a centrosome protein, Cep55, is required for its recruitment to midbody and cytokinesis. Dev Cell 9: 477-488, 2005.

9. Morita E, Sandrin V, Chung HY, Morham SG, Gygi SP, Rodesch CK and Sundquist WI: Human ESCRT and ALIX proteins interact with proteins of the midbody and function in cytokinesis. EMBO J 26: 4215-4227, 2007.

10. Carlton JG and Martin-Serrano J: Parallels between cytokinesis and retroviral budding: A role for the ESCRT machinery. Science 316: 1908-1912, 2007.

11. Sakai M, Shimokawa T, Kobayashi T, Matsushima S, Yamada Y, Nakamura Y and Furukawa Y: Elevated expression of C10orf3 (chromosome 10 open reading frame 3 ) is involved in the growth of human colon tumor. Oncogene 25: 480-486, 2006.

12. Chen CH, Lai JM, Chou TY, Chen CY, Su LJ, Lee YC, Cheng TS, Hong YR, Chou CK, Whang-Peng J, et al: VEGFA upregulates FLJ10540 and modulates migration and invasion of lung cancer via PI3K/AKT pathway. PLoS One 4: e5052, 2009.

13. Chen CH, Lu PJ, Chen YC, Fu SL, Wu KJ, Tsou AP, Lee YC, Lin TC, Hsu SL, Lin WJ, et al: FLJ10540-elicited cell transformation is through the activation of PI3-kinase/AKT pathway. Oncogene 26: 4272-4283, 2007.

14. FIGO Committee on Gynecologic Oncology: FIGO staging for carcinoma of the vulva, cervix, and corpus uteri. Int J Gynaecol Obstet 125: 97-98, 2014

15. Livak KJ and Schmittgen TD: Analysis of relative gene expression data using real-time quantitative PCR and the 2(-Delta Delta C(T)) method. Methods 25: 402-408, 2001.

16. Marth C, Landoni F, Mahner S, McCormack M, Gonzalez-Martin A and Colombo N; ESMO Guidelines Committee: Cervical cancer: ESMO clinical practice guidelines for diagnosis, treatment and follow-up. Ann Oncol 28: iv72-iv83, 2017.

17. Nigg EA and Raff JW: Centrioles, centrosomes, and cilia in health and disease. Cell 139: 663-678, 2009.

18. Nakamura M, Masuda H, Horii J, Kuma Ki, Yokoyama N, Ohba T, Nishitani H, Miyata T, Tanaka M and Nishimoto T: When overexpressed, a novel centrosomal protein, RanBPM, causes ectopic microtubule nucleation similar to gamma-tubulin. J Cell Biol 143: 1041-1052, 1998. 
19. Zhang W, Niu C, He W, Hou T, Sun X, Xu L and Zhang Y: Upregulation of centrosomal protein 55 is associated with unfavorable prognosis and tumor invasion in epithelial ovarian carcinoma. Tumour Biol 37: 6239-6254, 2016.

20. Chang YC, Wu CH, Yen TC and Ouyang P: Centrosomal protein 55 (Cep55) stability is negatively regulated by p53 protein through Polo-like kinase 1 (Plk1). J Biol Chem 287: 4376-4385, 2012.

21. Tao J, Zhi X, Tian Y, Li Z, Zhu Y, Wang W, Xie K, Tang J, Zhang X, Wang L, Xu Z: CEP55 contributes to human gastric carcinoma by regulating cell proliferation. Tumour Biol 35: 4389-4399, 2014.

22. Chen $\mathrm{CH}$, Chien $\mathrm{CY}$, Huang CC, Hwang CF, Chuang HC Fang FM, Huang HY, Chen CM, Liu HL and Huang CY: Expression of FLJ10540 is correlated with aggressiveness of oral cavity squamous cell carcinoma by stimulating cell migration and invasion through increased FOXM1 and MMP-2 activity. Oncogene 28: 2723-2737, 2009.
23. Chen CH, Shiu LY, Su LJ, Huang CY, Huang SC, Huang CC, Yin YF, Wang WS, Tsai HT, Fang FM, et al: FLJ10540 is associated with tumor progression in nasopharyngeal carcinomas and contributes to nasopharyngeal cell proliferation, and metastasis via osteopontin/CD44 pathway. J Transl Med 10: 93, 2012.

24. Hwang CF, Shiu LY, Su LJ, Yu-Fang Yin, Wang WS, Huang SC, Chiu TJ, Huang CC, Zhen YY, Tsai HT, et al: Oncogenic fibulin-5 promotes nasopharyngeal carcinoma cell metastasis through the FLJ10540/AKT pathway and correlates with poor prognosis. PLoS One 8: e84218, 2013. 